\title{
ON GROWTH OF DOUBLE COSETS IN HYPERBOLIC GROUPS
}

\author{
RITA GITIK AND ELIYAHU RIPS
}

\begin{abstract}
Let $H$ be a hyperbolic group, $A$ and $B$ be subgroups of $H$, and $\operatorname{gr}(H, A, B)$ be the growth function of the double cosets $A h B, h \in H$. We prove that the behavior of $\operatorname{gr}(H, A, B)$ splits into two different cases. If $A$ and $B$ are not quasiconvex, we obtain that every growth function of a finitely presented group can appear as $\operatorname{gr}(H, A, B)$. We can even take $A=B$. In contrast, for quasiconvex subgroups $\mathrm{A}$ and $\mathrm{B}$ of infinite index, $\operatorname{gr}(H, A, B)$ is exponential. Moreover, there exists a constant $\lambda>0$, such that $\operatorname{gr}(H, A, B)(r)>\lambda f_{H}(r)$ for all big enough $r$, where $f_{H}(r)$ is the growth function of the group $H$. So, we have a clear dychotomy between the quasiconvex and non-quasiconvex case.
\end{abstract}

\section{INTRODUCTION}

Growth of groups has been a subject of research for many years. For main results and references see [7. Growth of cosets and related subsets in groups has also been investigated, see for example [9] and [3. However, de la Harp wrote in [7, p.209 that growth of double cosets in groups has not yet received much attention, but probably should. In this paper we investigate growth of double cosets of hyperbolic groups.

Let $H$ be a hyperbolic group and let $A$ and $B$ be finitely generated subgroups of $H$. Fix some set of generators of $H$. For any $k \geq 0$, let $\operatorname{gr}(H, A, B)(r)$ be the growth function for double cosets $A h B$, that is $\operatorname{gr}(H, A, B)(r)=|\{A h B,|h| \leq r\}|$, where $|h|$ is the length of $h$.

Our first theorem shows that the class of growth rate functions of double cosets of non-quasiconvex subgroups is rather wide. Namely, let $G=\left\langle x_{1}, \cdots, x_{m} \mid r_{1}, \cdots, r_{n}\right\rangle$ be any finitely presented group. Let $f_{G}$ be the growth function of $G$, that is $f_{G}(r)=|\{g \in G,|g| \leq r\}|$, where the length $|g|$ is taken with respect to the set of generators $x_{1}, \cdots, x_{m}$.

Theorem 1. There exists a hyperbolic group $H$ and a finitely generated subgroup $N$ of $H$ such that $\operatorname{gr}(H, N, N)=f_{G}$.

Our second theorem shows that if $A$ and $B$ are quasiconvex then the growth function of the double cosets $\operatorname{gr}(H, A, B)$ is exponential.

Theorem 2. Let $H$ be a non-elementary hyperbolic group with a fixed set of generators. Let $A$ and $B$ be quasiconvex subgroups of $H$ of infinite index. Then there exists a constant $\lambda>0$ such that $\operatorname{gr}(H, A, B)(r) \geq \lambda f_{H}(r)$ for all big enough $r$.

Date: August 5, 2019.

2010 Mathematics Subject Classification. Primary: 20F67; Secondary: 20F65, $20 B 07$. 


\section{Proof of The First Theorem}

Let $G=\left\langle x_{1}, \cdots, x_{m} \mid r_{1}, \cdots, r_{n}\right\rangle$ be a finitely presented group. By a well-known construction of the second author [10], there exists a hyperbolic group $H$ and a short exact sequence $1 \longrightarrow N \stackrel{\alpha}{\longrightarrow} H \stackrel{\beta}{\longrightarrow} G \longrightarrow 1$ such that the normal subgroup $N$ of $H$ is finitely generated as a group. Indeed, following [10, define $H$ to be a group generated by the elements $x_{1}, \cdots, x_{m}, t_{1}, t_{2}$ with defining relations

$r_{i} t_{1} t_{2}^{a_{i}} t_{1} t_{2}^{a_{i}+1} \cdots t_{1} t_{2}^{b_{i}} \quad(i=1,2, \cdots, n)$,

$x_{i}^{-1} t_{j} x_{i} t_{1} t_{2}^{c_{i j}} t_{1} t_{2}^{c_{i j+1}} \cdots t_{1} t_{2}^{d_{i j}} \quad(i=1,2, \cdots, m, j=1,2)$,

$x_{i} t_{j} x_{i}^{-1} t_{1} t_{2}^{e_{i j}} t_{1} t_{2}^{e_{i j+1}} \cdots t_{1} t_{2}^{f_{i j}} \quad(i=1,2, \cdots, m, j=1,2)$.

We can choose the constants $a_{i}, b_{i}, c_{i j}, d_{i j}, e_{i j}, f_{i j}$ such that $H$ is a small cancellation group with arbitrarily small constant, and hence $H$ is a hyperbolic group.

Let us show that $\operatorname{gr}(H, N, N)=f_{G}$.

For every double coset $N h N$ consider the element $\beta(h) \in G$. This is a 1-to-1 correspondence between double cosets and elements of $G$. Indeed, $N h_{1} N=N h_{2} N$ implies $\beta\left(h_{1}\right)=\beta\left(h_{2}\right)$. Also for every $g=x_{i_{1}}^{\epsilon_{1}} x_{i_{2}}^{\epsilon_{2}} \cdots x_{i_{s}}^{\epsilon_{s}} \in G, \beta(g)=g$, so to $N g N$ corresponds the element $\beta(g)=g \in G$.

The homomorphism $\beta: H \longrightarrow G$ sends $x_{i}$ to $x_{i}$ and $t_{j}$ to 1 for $i=1,2, \cdots, m, j=$ 1,2. It follows that for every $h \in H,|\beta(h)| \leq|h|$, where the length of $h$ is taken with respect to the generators $x_{1}, \cdots, x_{m}, t_{1}, t_{2}$ of $H$ and the length of $\beta(h)$ is taken with respect to the generators $x_{1}, \cdots, x_{m}$ of $G$.

Therefore, for any $k \geq 0$, the number of double cosets $N h N$ with $|h| \leq k$ is equal to the number of elements $g \in G$ with $|g| \leq k$. Hence, $\operatorname{gr}(H, N, N)=f_{G}$, proving Theorem 1.

\section{Proof of The Second Theorem}

Claim 1. Let $A$ be an infinite index quasiconvex subgroup of a hyperbolic group $H$. There exists an element $c_{1} \in H$ of infinite order such that $A \cap\left\langle c_{1}\right\rangle=\{1\}$.

Proof. Denote the boundary of $H$ by $\partial H$. Note that the limit set $\Lambda(A)$ of $A$ in $\partial H$ is a closed subset. As $A$ is infinite and quasiconvex, it has finite index in the setwise stabilizer $\operatorname{Stab}_{H}(\Lambda(A))$. In particular, this implies that $\Lambda(A) \neq \partial H$ because if $\Lambda(A)=\partial H$ then $\operatorname{Stab}_{H}(\Lambda(A))=H$, contradicting the assumption that $A$ has infinite index in $H$. Hence $\Omega=\partial H-\Lambda(A)$ is a nonempty open subset of $\partial H$.

Since $H$ is hyperbolic, the set of poles $t_{+}$of all infinite order elements $t \in H$ is dense in $\partial H$. Hence there exists an element $c_{1} \in H$ of infinite order such that $c_{1+} \in \Omega$. Therefore $A \cap\left\langle c_{1}\right\rangle=\{1\}$, since otherwise $c_{1}^{n} \in A$ for some $n>0$ and $c_{1}+\in \Lambda(A)$, contradicting the assumption that $c_{1}+\in$.

The only facts used in the proof of Claim 1 are:

(1) Poles of infinite order elements are dense in the boundary of the group $H$.

(2) If $A$ is an infinite quasiconvex subgroup of $H$ then $A$ has finite index in $\operatorname{Stab}_{H}(\Lambda(A))$.

The aforementioned facts hold for an arbitrary hyperbolic group $H$, without any restrictions on the existence of nontrivial torsion elements.

Claim 2. Let $H$ be a non-elementary hyperbolic group, let $A$ be a quasiconvex subgroup of infinite index in $H$, and let $c_{1} \in H$ be an element of infinite order such that $A \cap\left\langle c_{1}\right\rangle=\{1\}$. Denote by $C_{1}$ the commensurator of $\left\langle c_{1}\right\rangle$ in $H$ and let 
$C_{2}=A \cap C_{1}$. There exists a large integer $M$ such that for $C=\left\langle c_{1}^{M}, C_{2}\right\rangle$ the following holds: $\langle A, C\rangle=\underset{C_{2}}{*} C$ and $\langle A, C\rangle$ is a quasiconvex subgroup of $H$.

Claim 2 follows from Theorem 1.1 in [8, which generalizes Theorem 2 in [4, because any non-elementary hyperbolic group $G$ is hyperbolic relative to the commensurator of an infinite cyclic subgroup. That was observed in Theorem 7.11 in [2].

Claim 3. Let $H$ be a non-elementary hyperbolic group with a fixed generating set and let $\left(\epsilon_{0}, \eta_{0}\right)$ be constants. There exist geodesic words $x$ and $y$, and constants $(\epsilon, \eta)$ such that for any $\left(\epsilon_{0}, \eta_{0}\right)$-quasigeodesic words $u$ and $v$ in $H$ at least one of the words $\left\{u x v, u x^{-1} v, u y v, u y^{-1} v\right\}$ is an $(\epsilon, \eta)$-quasigeodesic.

Proof. As we are interested only in the existence of the constants $(\epsilon, \eta)$ we do not have to compute them explicitly. Let $\delta$ be a hyperbolicity constant of $H$. Denote the Gromov product of the words $w$ and $z$ by $(w, z)_{1}$, cf. [6] and [1] definition 2.6.

For a big enough constant $C_{0}$ there exist geodesic words $x$ and $y$ which are longer than $C_{0}$, such that $(w, z)_{1} \leq \beta$, where $\beta$ is a constant bigger than $\delta$, and the words $w$ and $z$ belong to the set $\left\{x, x^{-1}, y, y^{-1}\right\}$.

Note that for any $\left(\epsilon_{0}, \eta_{0}\right)$-quasigeodesic $u$ only one of the Gromov products $\left\{(u, x)_{1},\left(u, x^{-1}\right)_{1},(u, y)_{1},\left(u, y^{-1}\right)_{1}\right\}$ might be big. Similarly, only one of the Gromov products $\left\{\left(v^{-1}, x\right)_{1},\left(v^{-1}, x^{-1}\right)_{1},\left(v^{-1}, y\right)_{1},\left(v^{-1}, y^{-1}\right)_{1}\right\}$ might be big. Therefore, at least one of the following conditions holds.

(1) $(u, x)_{1}$ and $\left(v^{-1}, x^{-1}\right)_{1}$ are small;

(2) $\left(u, x^{-1}\right)_{1}$ and $\left(v^{-1}, x\right)_{1}$ are small;

(3) $(u, y)_{1}$ and $\left(v^{-1}, y^{-1}\right)_{1}$ are small;

(4) $\left(u, y^{-1}\right)_{1}$ and $\left(v^{-1}, y\right)_{1}$ are small.

In case (1) $u x v$ is a quasigeodesic, in case (2) $u x^{-1} v$ is a quasigeodesic, in case (3) $u y v$ is a quasigeodesic, in case (4) $u y^{-1} v$ is a quasigeodesic. Note that the quasigeodesicity constants $(\epsilon, \eta)$ do not depend on $u$ and $v$.

\section{Proof of Theorem 2.}

Since $A$ and $B$ are quasiconvex and of infinite index in $H$, Claim 1 implies that there exist elements $c_{1} \in H$ and $d_{1} \in H$ of infinite order such that $\left\langle c_{1}\right\rangle \cap A=\{1\}$ and $\left\langle d_{1}\right\rangle \cap B=\{1\}$. Denote by $C_{1}$ the commensurator of $\left\langle c_{1}\right\rangle$ in $H$ and by $D_{1}$ the commensurator of $\left\langle d_{1}\right\rangle$ in $H$. Let $C_{2}=C_{1} \cap A$ and $D_{2}=D_{1} \cap B$. Claim 2 implies that there exists a large integer $M$ such that the subgroups $C=\left\langle c_{1}^{M}, C_{2}\right\rangle$ and $D=\left\langle d_{1}^{M}, D_{2}\right\rangle$ have the following properties: $\langle A, C\rangle=A_{C_{2}}^{*} C$ and $\langle B, D\rangle=B \underset{D_{2}}{*} D$. Denote $c=c_{1}^{M}$ and $d=d_{1}^{M}$.

According to Claim 3, there exists a large integer $N$ such that for a long enough geodesic word $t$ there exist $z$ and $w$ in the set $\left\{x, x^{-1}, y, y^{-1}\right\}$ such that $s=c^{N} z t w d^{N}$ is $(\epsilon, \eta)$-quasigeodesic. Note that $|s| \leq N|c|+N|d|+|z|+|w|+|t| \leq$ $N(|c|+|d|)+2|x|+2|y|+|t|$. So if $|t| \leq r-(N(|c|+|d|)+2|x|+2|y|+|t|)$ then $|s| \leq r$. As $w$ and $z$ belong to the set $\left\{x, x^{-1}, y, y^{-1}\right\}$, no more than 16 different choices of $t$ can produce the same word $s$.

Let $m=f_{H}\left(r-N(|c|+|d|)+2|x|+2|y|-f_{H}\left(C_{0}\right)\right)$. There exist geodesic words $t_{1}, t_{2}, \cdots, t_{m}$ such that $C_{0}<\left|t_{i}\right| \leq r-N(|c|+|d|+2|x|+2|y|)$ for $1 \leq i \leq m$. It follows that the corresponding words $s_{i}=c^{N} z_{i} t_{i} w_{i} d^{N}$ are $(\epsilon, \eta)$-quasigeodesics 
and $z_{i}$ and $w_{i}$ belong to the set $\left\{x, x^{-1}, y, y^{-1}\right\}$. As $f_{H}$ is the growth function of a non-elementary hyperbolic group, there exists a positive constant $\mu$ such that $16 f_{H}(r-(N(|c|+|d|)+2|x|+2|y|)) \geq \mu f_{H}(r)$.

Consider the double cosets $\left\{A s_{i} B \mid 1 \leq i \leq m\right\}$. If $A s_{i} B=A s_{j} B, i \neq j$, then there exist geodesic words $a \in A$ and $b \in B$ such that $s_{j}={ }_{H} a s_{i} b$ in $H$, hence $b={ }_{H} s_{i}^{-1} a^{-1} s_{j}={ }_{H} d^{-N} w_{i}^{-1} t_{i}^{-1} z_{i}^{-1} c^{-N} a c^{N} z_{j} t_{j} w_{j} d^{N}$.

If $a \notin C_{2}$ then, according to Theorem 1.1 of [8], the word $c^{-N} a^{-1} c^{N}$ is a quasigeodesic. As $d^{-N} w_{i}^{-1} t_{i}^{-1} z_{i}^{-1} c_{0}^{-N}$ and $c^{N} z_{j} t_{j} w_{j} d^{N}$ are quasigeodesics, it follows that $d^{-N} w_{i}^{-1} t_{i}^{-1} z_{i}^{-1} c^{-N} a^{-1} c^{N} z_{j} t_{j} w_{j} d^{N}$ is also a quasigeodesic, contradicting the fact that it is equal to $b \in B$ in $H$, where the $\operatorname{subgroup} B$ is quasiconvex. Similarly, it cannot happen that $b \notin D_{2}$. Hence $s_{j}={ }_{H} a s_{i} b$ implies that $a \in C_{2}$ and $b \in D_{2}$.

Therefore at least $\frac{m}{\left|C_{2}\right| \cdot\left|D_{2}\right|}$ of the double cosets $A s_{i} B, i=1, \cdots, m$ are different from each other. So $\operatorname{gr}(H, A, B)(r) \geq \frac{\mu}{\left|C_{2}\right| \cdot\left|D_{2}\right|} \cdot f_{H}(r)$ and $\lambda=\frac{\mu}{\left|C_{2}\right| \cdot\left|D_{2}\right|}$ satisfies the requirements of Theorem 2 .

\section{Corollary to the Proof of Theorem 2.}

Using the notation of the Proof of Theorem 2, for each $i=1,2, \ldots, m$ the subgroup $\left\langle A, s_{i} B s_{i}^{-1}\right\rangle$ is a quasiconvex subgroup of $H$, and its geodesic core (see Definition 4 in 5]) consists of a cylindrical neighborhood of the path for $s_{i}$ with geodesic cores of $A$ and of $B$ attached at the beginning and at the end of the path for $s_{i}$ respectively.

\section{ACKNowledgment}

We would like to thank Eduardo Martinez-Pedroza for bringing to our attention his paper 8 and to Ilya Kapovich for helpful discussions.

\section{REFERENCES}

[1] N. Benakli and I. Kapovich, Boundaries of Hyperbolic Groups, Comtemporary Math., 296(2002), 39-93, AMS, Providence.

[2] B. H. Bowditch, Relatively Hyperbolic Groups, IJAC, 22(2012), 1250016.

[3] M. Coornaet and G. Knieper, Growth of Conjugacy Classes in Gromov Hyperbolic Groups, GAFA, 12(2002), 464-478.

[4] R. Gitik, Ping-Pong on Negatively Curved Groups, J. of Algebra, 217(1999), 65-72.

[5] R. Gitik, Tameness and Geodesic Cores of Subgroups, J. Austral. Math. Soc. (Series A), 69(2000), 153-161.

[6] M. Gromov, Hyperbolic Groups, Essays in Group Theory, MSRI Publications, 8(1987), 75263, Springer, New York.

[7] P. de la Harpe, Topics in Geometric Group Theory, Chicago Lectures in Mathematics, The University of Chicago Press, 2000.

[8] E. Martinez-Pedroza, Combination of Quasiconvex Subgroups of Relatively Hyperbolic Groups, Groups Geom. Dyn., 3(2009), 317342.

[9] A. Olshanskii, Subnormal Subgroups in Free Groups, Their Growth And Cogrowth, Math. Proc. Cambridge Phil. Soc., 163(2017), 499-531.

[10] E. Rips, Subgroups of Small Cancellation Groups, Bull. LMS, 14(1982), 45-47.

Department of Mathematics, University of Michigan, Ann Arbor, Mi, 48109

E-mail address: ritagtk@umich.edu

Institute of Mathematics, Hebrew University, Jerusalem, 91904, Israel

E-mail address: eliyahu.rips@mail.huji.ac.il 